\title{
Transport through a quantum wire with a side quantum-dot array
}

\author{
P. A. Orellana, ${ }^{1}$ F. Domínguez-Adame, ${ }^{2}$ I. Gómez, ${ }^{2}$ and M. L. Ladrón de Guevara ${ }^{3}$ \\ ${ }^{1}$ Departamento de Física, Universidad Católica del Norte, Casilla 1280, Antofagasta, Chile \\ ${ }^{2}$ GISC, Departamento de Física de Materiales, Universidad Complutense, E-28040 Madrid, Spain \\ ${ }^{3}$ Departamento de Física, P. Universidad Católica de Chile, Casilla 306, Santiago 22, Chile
}

(Received 26 October 2002; published 28 February 2003)

\begin{abstract}
A noninteracting quantum-dot array side coupled to a quantum wire is studied. Transport through the quantum wire is investigated by using a noninteracting Anderson tunneling Hamiltonian. The conductance at zero temperature develops an oscillating band with resonances and antiresonances due to constructive and destructive interference in the ballistic channel, respectively. Moreover, we have found an odd-even parity in the system, whose conductance vanishes for an odd number of quantum dots while it becomes $2 e^{2} / h$ for an even number. We established an explicit relation between this odd-even parity and the positions of the resonances and antiresonances of the conductivity with the spectrum of the isolated quantum-dot array.
\end{abstract}

DOI: 10.1103/PhysRevB.67.085321

PACS number(s): 73.21.La, 73.63.Kv, 85.35.Be

\section{INTRODUCTION}

Recent progress in the nanofabrication of quantum devices has enabled us to study electron transport through quantum dots (QD's) in a very controllable way. ${ }^{1,2}$ QD's are very promising systems due to their physical properties as well as their potential applications in electronic devices. These structures are small semiconductor or metal structures in which electrons are confined in all spatial dimensions. As a consequence, discreteness of the energy and charge arises. For this reason QD's are often referred as artificial atoms. In contrast to real atoms, different regimes can be studied by continuously changing the applied external potential.

If the single QD is referred to as artificial atom, a QD array can be considered as an artificial molecule or artificial crystal. ${ }^{3-5}$ The latest advances in nanotechnology make it possible to fabricate QD arrays. In linear QD arrays leads are attached to their ends and the current through them is measured while external parameters such a gated voltage, magnetic field, and temperature are varied. In the resonant tunneling regime, electronic transport through the QD array becomes sensitive to a precise matching of the electron levels in the dots that can be controlled experimentally. On the other hand, a linear QD array can be seen as a onedimensional chain of sites. This type of chain coupled to the continuum states shows an even-odd parity effect in the conductance when the Fermi energy is localized in the center of the energy band. The conductance is $2 e^{2} / h$ for odd samples and is smaller for even parity. ${ }^{6-8}$

The aim of this work is to study theoretically the transport properties of an alternative configuration of a side-coupled QD array attached to a perfect quantum wire (QW). In this case the QD array acts as a scattering center for transmission through the QW. This configuration can be regarded as a quantum wave guide with side-stub structures, similar to those reported in Refs. 9-11. In contrast to the embedded QD array, the transmission through the side-coupled QD array consists of the interference between the ballistic channel and the resonant channels from the QD array. For a uniform side QD array, we found that the system develops an oscillating band with resonances (perfect transmission) and anti- resonances (perfect reflection). In addition, we found an oddeven parity of the number of QD's in the array: namely, perfect transmission takes place if this number is even $(G$ $\left.=2 e^{2} / h\right)$ but perfect reflection arises for an odd number $(G=0)$. This result is opposed to that found in embedded QD arrays. ${ }^{6-8}$ We established an explicit relation between this odd-even parity and the positions of the resonances and antiresonances of the conductivity with the spectrum of the isolated QD array.

\section{MODEL}

Let us consider a QW with a side-coupled QD array. The array consists of $N$ QD's connected in a series by tunnel coupling, as shown in Fig. 1. The system is modeled by using a noninteracting Anderson tunneling Hamiltonian, ${ }^{5}$ which can be written as

$$
H=H_{\mathrm{QW}}+H_{\mathrm{QD}}^{N}+H_{\mathrm{QD}-\mathrm{QW}},
$$

with

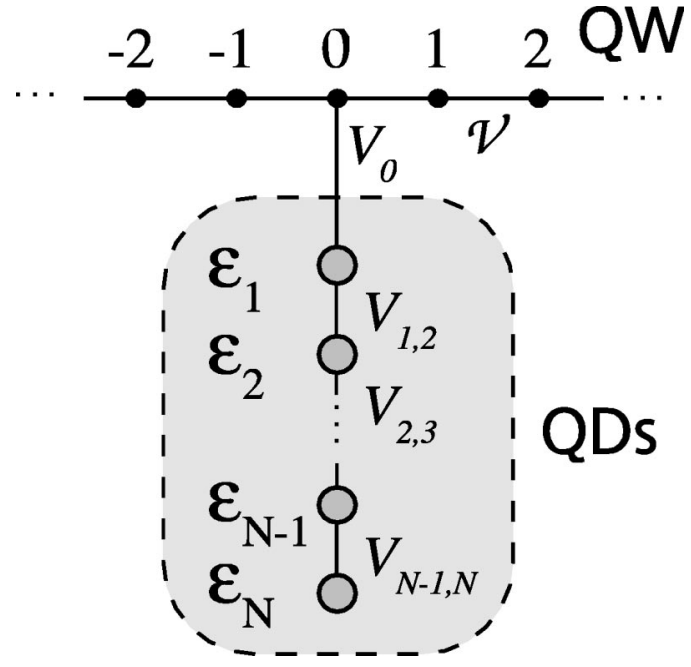

FIG. 1. Side-coupled quantum-dot array attached to a perfect quantum wire. 


$$
\begin{gathered}
H_{\mathrm{QW}}=v \sum_{i \neq j} c_{i}^{\dagger} c_{j}, \\
H_{\mathrm{QD}}^{N}=\sum_{l=1}^{N} \varepsilon_{l} d_{l}^{\dagger} d_{l}+\sum_{l=1}^{N-1}\left(V_{l, l+1} d_{l}^{\dagger} d_{l+1}+\text { H.c. }\right), \\
H_{\mathrm{QD}-\mathrm{QW}}=V_{0}\left(d_{1}^{\dagger} c_{0}+c_{0}^{\dagger} d_{1}\right) .
\end{gathered}
$$

The operator $c_{i}^{\dagger}$ creates an electron at site $i, v$ is the hopping in the QW, $\varepsilon_{l}$ is the energy level of the dot $l$, and $V_{l, l+1}$ is the tunneling coupling between the $l$ th and $(l+1)$ th QD. Here $H_{\mathrm{QW}}$ corresponds to the free-particle Hamiltonian on a lattice with spacing $d$ and whose eigenfunctions are expressed as Bloch solutions

$$
|k\rangle=\sum_{j=-\infty}^{\infty} e^{i k d j}|j\rangle,
$$

where $|k\rangle$ is the momentum eigenstate and $|j\rangle$ is a Wannier state localized at site $j$. The dispersion relation associated with these Bloch states reads

$$
\varepsilon=2 v \cos (k d) .
$$

Consequently, the Hamiltonian supports an energy band from $-2 v$ to $+2 v$ and the first Brillouin zone expands the interval $[-\pi / d, \pi / d]$.

The stationary states of the entire Hamiltonian $H$ can be written as

$$
\left|\psi_{k}\right\rangle=\sum_{j=-\infty}^{\infty} a_{j}^{k}|j\rangle+\sum_{l=1}^{N} b_{l}^{k}|l\rangle
$$

where the coefficient $a_{j}^{k}\left(b_{l}^{k}\right)$ is the probability amplitude to find the electron in the site $j$ of the QW ( $l$ of the array) in the state $k$, namely,

$$
a_{j}^{k}=\left\langle j \mid \psi_{k}\right\rangle, \quad b_{l}^{k}=\left\langle l \mid \psi_{k}\right\rangle .
$$

The amplitudes $a_{j}^{k}$ obey the linear difference equations

$$
\begin{gathered}
\varepsilon a_{j}^{k}=v\left(a_{j-1}^{k}+a_{j+1}^{k}\right)+V_{0} b_{1}^{k} \delta_{j 0}, \\
\varepsilon b_{1}^{k}=\varepsilon_{1} b_{1}^{k}+V_{1,2} b_{2}^{k}+V_{0} a_{0}^{k}, \\
\varepsilon b_{l}^{k}=\varepsilon_{l} b_{l}^{k}+V_{l, l-1} b_{l-1}^{k}+V_{l, l+1} b_{l+1}^{k}, \quad l \neq 1, N, \\
\varepsilon b_{N}^{k}=\varepsilon_{N} b_{N}^{k}+V_{N, N-1} b_{N-1}^{k} .
\end{gathered}
$$

Iterating backwards the equation for $b_{N}^{k}$ we can express the amplitude $b_{1}^{k}$ in terms of $a_{0}^{k}$ as a continued fraction

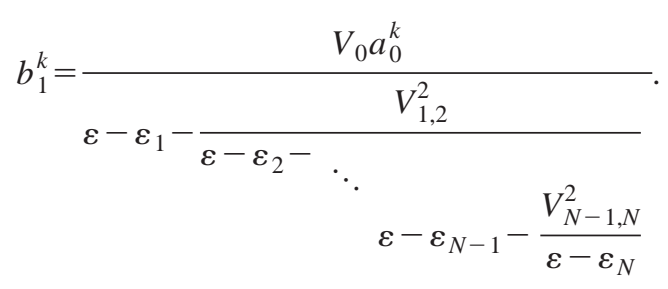

Therefore the equation for $a_{0}^{k}$ can be cast in the form

$$
\varepsilon a_{0}^{k}=v\left(a_{-1}^{k}+a_{1}^{k}\right)+V_{0}^{2} / Q_{N} a_{0}^{k},
$$

where $Q_{N}$ is the continued fraction

$$
Q_{N}=\varepsilon-\varepsilon_{1}-\frac{V_{1,2}^{2}}{\varepsilon-\varepsilon_{2}-} \begin{aligned}
& \quad \\
& \varepsilon-\varepsilon_{N-1}-\frac{V_{N-1, N}^{2}}{\varepsilon-\varepsilon_{N}}
\end{aligned} .
$$

In order to study the solutions of Eqs. (7) we assume that the electrons are described by a plane wave incident from the far left with unity amplitude and a reflection amplitude $r$ and at the far right by a transmission amplitude $t$. Taking this to be the solution we can write

$$
\begin{gathered}
a_{j}^{k}=e^{i k d j}+r e^{-i k d j}, \quad j<0, \\
a_{j}^{k}=t e^{i k d j}, \quad j>1 .
\end{gathered}
$$

The solution of the equations for the $a_{j}^{k}$ s can be then obtained iteratively from right to left. For a given transmission amplitude, the associated incident and reflection amplitudes may be determined by matching the iterated function to the proper plane wave at the far left. The transmission probability is given by $T=|t|^{2}$ and is obtained from the iterative procedure described above. In equilibrium we solve the equation for $t$ and $r$ and we get the expressions

$$
\begin{gathered}
t=\frac{2 i v \sin (k d)}{2 i v \sin (k d)-V_{0}^{2} / Q_{N}}=\frac{Q_{N}}{Q_{N}+i \Gamma}, \\
r=-\frac{V_{0}^{2} / Q_{N}}{2 i v \sin (k d)-V_{0}^{2} / Q_{N}}=\frac{i \Gamma}{Q_{N}+i \Gamma},
\end{gathered}
$$

where $\Gamma(\varepsilon) \equiv V_{0}^{2} / 2 v \sin (k d)$ can be regarded as the level broadening. Notice that the level broadening can be fairly well approximated by $\Gamma \simeq V_{0}^{2} / 2 v$ close to the center of the band.

The experimentally accessible quantity is the linear conductance $G$ which is related to the transmission coefficient $T$ at the Fermi energy by the one-channel Landauer formula at zero temperature:

$$
G=\frac{2 e^{2}}{h} T=\frac{2 e^{2}}{h} \frac{Q_{N}^{2}}{Q_{N}^{2}+\Gamma^{2}} .
$$

It is worth mentioning that the energy levels (zeros of $\left.Q_{N}\right)$ depend only on the hopping in the QD array $\left(V_{n-1, n}\right)$ while $\Gamma$ is the only function of $V_{0}^{2} / v$. Consequently, both magnitudes can be controlled independently in an actual experiment. This is one of the main advantages of the present setup. 


\section{RESULTS}

\section{A. Short QD array}

Closed expressions for the transmission and reflection coefficients can be readily obtained when the number of QD's in the array is small. For $N=1, Q_{1}=\varepsilon-\varepsilon_{1}$ and then we arrive at

$$
\begin{aligned}
& T(\varepsilon)=\frac{\left(\varepsilon-\varepsilon_{1}\right)^{2}}{\left(\varepsilon-\varepsilon_{1}\right)^{2}+\Gamma^{2}}, \\
& R(\varepsilon)=\frac{\Gamma^{2}}{\left(\varepsilon-\varepsilon_{1}\right)^{2}+\Gamma^{2}} .
\end{aligned}
$$

The system has an antiresonance at $\varepsilon=\varepsilon_{1}$. The transmission and the reflection probability are 0 and 1 , respectively. For $N=2$, the transmission and reflection coefficients are given by

$$
\begin{aligned}
& T(\varepsilon)=\frac{\left[\left(\varepsilon-\varepsilon_{1}\right)\left(\varepsilon-\varepsilon_{2}\right)-V_{c}^{2}\right]^{2}}{\left[\left(\varepsilon-\varepsilon_{1}\right)\left(\varepsilon-\varepsilon_{2}\right)-V_{c}^{2}\right]^{2}+\left(\varepsilon-\varepsilon_{2}\right)^{2} \Gamma^{2}}, \\
& R(\varepsilon)=\frac{\left(\varepsilon-\varepsilon_{2}\right)^{2} \Gamma^{2}}{\left[\left(\varepsilon-\varepsilon_{1}\right)\left(\varepsilon-\varepsilon_{2}\right)-V_{c}^{2}\right]^{2}+\left(\varepsilon-\varepsilon_{2}\right)^{2} \Gamma^{2}},
\end{aligned}
$$

where $V_{1,2} \equiv V_{c}$. Therefore, the system presents one resonance in $\varepsilon-\varepsilon_{2}$ and bonding and antibonding antiresonances at energies

$$
\varepsilon=\frac{1}{2}\left(\varepsilon_{1}+\varepsilon_{2}\right) \pm \frac{1}{2} \sqrt{\left(\varepsilon_{1}-\varepsilon_{2}\right)^{2}+4 V_{c}^{2}} .
$$

The system with $N=3$ side-coupled QD's shows particularly simple solution for the case $V_{1,2}=V_{2,3} \equiv V_{c}$,

$$
\begin{gathered}
T(\varepsilon)=\frac{\left\{\left(\varepsilon-\varepsilon_{1}\right)\left(\varepsilon-\varepsilon_{2}\right)\left(\varepsilon-\varepsilon_{3}\right)-V_{c}^{2}\left[\left(\varepsilon-\varepsilon_{1}\right)+\left(\varepsilon-\varepsilon_{3}\right)\right]\right\}^{2}}{\left\{\left(\varepsilon-\varepsilon_{1}\right)\left(\varepsilon-\varepsilon_{2}\right)\left(\varepsilon-\varepsilon_{3}\right)-V_{c}^{2}\left[\left(\varepsilon-\varepsilon_{1}\right)+\left(\varepsilon-\varepsilon_{3}\right)\right]\right\}^{2}+\left[\left(\varepsilon-\varepsilon_{2}\right)\left(\varepsilon-\varepsilon_{3}\right)-V_{c}^{2}\right]^{2} \Gamma^{2}}, \\
R(\varepsilon)=\frac{\left[\left(\varepsilon-\varepsilon_{2}\right)\left(\varepsilon-\varepsilon_{3}\right)-V_{c}^{2}\right]^{2} \Gamma^{2}}{\left\{\left(\varepsilon-\varepsilon_{1}\right)\left(\varepsilon-\varepsilon_{2}\right)\left(\varepsilon-\varepsilon_{3}\right)-V_{c}^{2}\left[\left(\varepsilon-\varepsilon_{1}\right)+\left(\varepsilon-\varepsilon_{3}\right)\right]\right\}^{2}+\left[\left(\varepsilon-\varepsilon_{2}\right)\left(\varepsilon-\varepsilon_{3}\right)-V_{c}^{2}\right]^{2} \Gamma^{2}} .
\end{gathered}
$$

Clearly, for the case with $\varepsilon_{1}=\varepsilon_{2}=\varepsilon_{3}$, the system shows three antiresonances at $\varepsilon=\varepsilon_{1}$ and $\varepsilon=\varepsilon_{1} \pm \sqrt{2} V_{c}$ and two resonances at $\varepsilon=\varepsilon_{1} \pm V_{c}$.

Figure 2 shows the conductance as a function of the Fermi energy of the incident electron for $\varepsilon_{i}=0(i=1, \ldots, N)$ and $V_{c}=\Gamma$. There exists only one narrow antiresonance in the case of $N=1 \mathrm{QD}$, and bonding, and antibonding antiresonances and one resonance in zero are clearly revealed for $N=2$. In addition, bonding and antibonding resonances

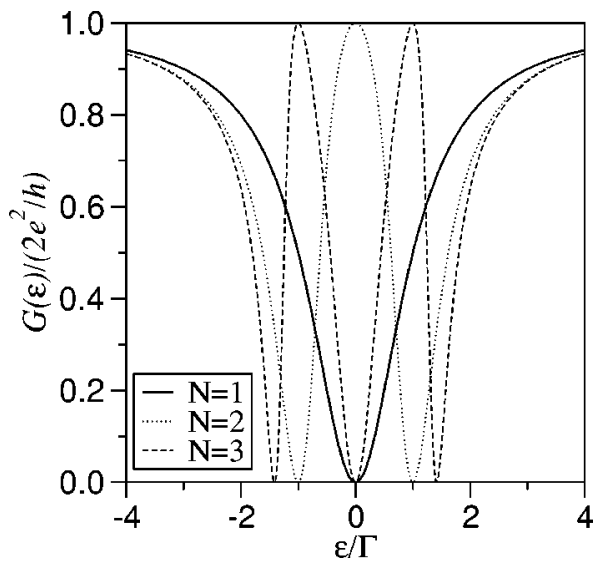

FIG. 2. Conductance, in units of $2 e^{2} / h$, vs Fermi energy, in units of the $\Gamma$, for the case of the one-, two-, and three-QD arrays with $V_{c}=\Gamma$. and zero, bonding and antibonding antiresonances arise when $N=3$.

\section{B. Long QD array}

When the number $N$ of attached QD's is large, we must rely on numerical calculations. For the sake of simplicity we consider a uniform quantum dot array $V_{l-1, l} \equiv V_{c}$ and $\varepsilon_{l}$ $=\varepsilon_{0}$. The continued fraction $Q_{N}$ in Eq. (10) is written as $Q_{N}=\left(\varepsilon-\varepsilon_{0}\right) x_{N}$, where $x_{N}$ satisfies the following recursive equation

$$
x_{N}=1-\frac{\alpha}{x_{N-1}}, \quad N=1,2,3, \ldots,
$$

with $x_{1}=1$ and $\alpha \equiv V_{c}^{2} /\left(\varepsilon-\varepsilon_{0}\right)^{2}$ for $\varepsilon \neq \varepsilon_{0}$.

For $N$ large the antiresonance appearing in Fig. 2 for $N$ $=1$ evolves as a fast oscillation band. As the number of QD's $N$, increases, this narrow antiresonance splits into $N$ antiresonances and $N-1$ resonances, as seen in the upper panel of Fig. 3 for $V_{c}=\Gamma$ and $\varepsilon_{0}=0$. On further increasing $N$, the antiresonances never merge into a single stop band, as one would naively expect. In fact, it is not difficult to demonstrate that $Q_{N}=D_{N} / D_{N-1}$, where $D_{N}=\operatorname{det}\left(H_{Q D}^{N}-\varepsilon I\right)$. The transmission coefficient can be written as

$$
T=\frac{D_{N}^{2}}{D_{N}^{2}+\Gamma^{2} D_{N-1}^{2}} .
$$




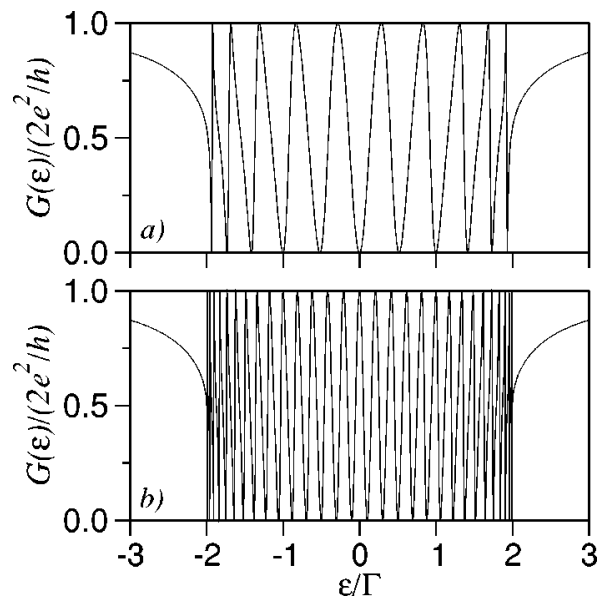

FIG. 3. Conductance, in units of $2 e^{2} / h$, vs Fermi energy, in units of $\Gamma$, for (a) $N=11$ and (b) $N=30$ QD arrays with $V_{c}=\Gamma$ and $\varepsilon_{0}=0$.

Thus, the transmission vanishes in the spectrum of $H_{Q D}^{N}$ and becomes unity in the spectrum of the $H_{Q D}^{N-1}$. Therefore the conductance shows $N$ antiresonances and $N-1$ resonances, as we see in Figs. 1 and 2. This statement is further confirmed by plotting the full width at half minimum of the antiresonances $\Delta$ as a function of energy, as shown in Fig. 4 for different values of $N$ and $\varepsilon_{0}=0$.

\section{Odd-even parity}

Here we consider the case when the Fermi energy is pinned at the value of the energy level of the quantum dot. From Eq. (7) is straightforward to prove the existence of an odd-even parity when $\varepsilon_{l}=\varepsilon_{0}$,

$$
\begin{gathered}
G=0, \quad N \text { odd, } \\
G=\frac{2 e^{2}}{h}, \quad N \text { even, }
\end{gathered}
$$

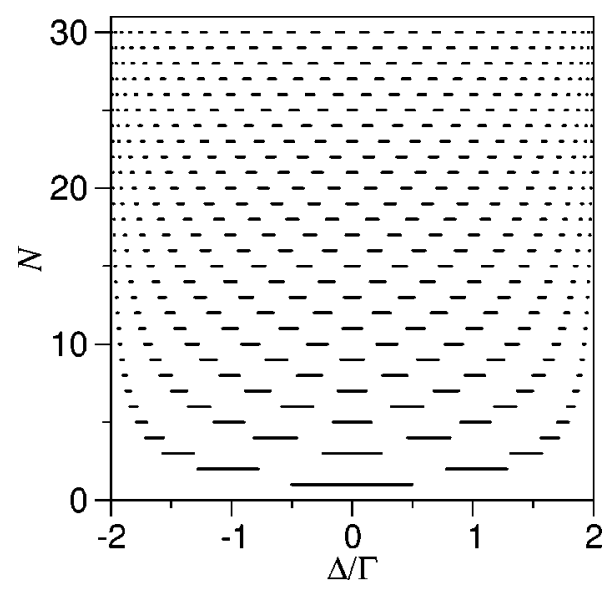

FIG. 4. Full width at half minimum of the antiresonances $\Delta$ for different values of $N$ and $V_{c}=\Gamma$. Each segment joints the two energies for which conductance becomes $e^{2} / h$ on every antiresonance. as can be readily checked in Figs. 1 and 2. This result also holds for QD arrays with off-diagonal disorder. This oddeven parity is opposed to the case of an embedded quantum array, where perfect transmission takes place for odd parity. ${ }^{6-8}$ This property arises from the fact that the energy level of the QD's, $\varepsilon_{0}$, is always in the electronic spectrum of the isolated QD array, provided the number of the QD's is odd. It is straightforward to demonstrate this statement from the fact $D_{N}$ satisfies the recursive equation

$$
D_{N}=\left(\varepsilon-\varepsilon_{0}\right) D_{N-1}-V_{N-1, N}^{2} D_{N-2}, \quad N=3,4,5, \ldots,
$$

with $D_{1}=\varepsilon-\varepsilon_{0}$ and $D_{2}=\left(\varepsilon-\varepsilon_{0}\right)^{2}-V_{1,2}^{2}$. It is clear from Eq. (21) that $D_{N}$ is zero at $\varepsilon=\varepsilon_{0}$ if $N$ is odd. Therefore $\varepsilon_{0}$ is an eigenvalue of $H_{Q D}^{N}$ and then from Eq. (19) we obtain that at $\varepsilon=\varepsilon_{0}, T=0$ (perfect reflection) if $N$ is odd and $T=1$ (perfect transmission) for even $N$.

\section{Infinite QD array}

To understand the origin of the fast oscillations of the conductance as a function of the Fermi energy (Fig. 3), we now consider the limiting case $N \rightarrow \infty$. Thus, we are faced with a one-dimensional map (18). This map has two fixed points at ${ }^{12}$

$$
x_{ \pm}^{*}=\frac{1}{2}(1 \pm \sqrt{1-4 \alpha}),
$$

when $\alpha<1 / 4$, namely, $\left|\varepsilon-\varepsilon_{0}\right|>2 V_{c}$. The conductance for $N \rightarrow \infty$ is

$$
G_{\infty}=\frac{2 e^{2}}{h} \frac{\left(\left|\varepsilon-\varepsilon_{0}\right|+\sqrt{\left(\varepsilon-\varepsilon_{0}\right)^{2}-4 V_{c}^{2}}\right)^{2}}{\left(\left|\varepsilon-\varepsilon_{0}\right|+\sqrt{\left.\left(\varepsilon-\varepsilon_{0}\right)^{2}-4 V_{c}^{2}\right)^{2}+\Gamma^{2}}\right.},
$$

for $\left|\varepsilon-\varepsilon_{0}\right|>2 V_{c}$. This result explains the smooth tails seen in Fig. 3 when $\left|\varepsilon-\varepsilon_{0}\right| / \Gamma>2$.

The conductance undergoes a bifurcation at $\alpha=1 / 4(\mid \varepsilon$ $-\varepsilon_{0} \mid=2 V_{c}$ ), and there are no fixed points when $\alpha>1 / 4$, namely, $\left|\varepsilon-\varepsilon_{0}\right|<2 V_{c}$. Consequently, minute variations of the Fermi energy result in a dramatic change in the conductance of the QW, as can be concluded from the lower panel of Fig. 3.

\section{SUMMARY}

In this work, we studied the conductance at zero temperature of a side QD array attached to a QW. For a uniform QD array we found that the system develops an oscillating band with $N$ antiresonances and $N-1$ resonances arising from the hybridization of the quasibound levels of the QD's and the coupling to the QW. The positions of the antiresonances correspond exactly to the electronic spectrum of the isolated QD array. This property could be used to measure the energy spectrum of the $N$ QD array. It should be stressed that the particular setup we suggested allows us to control the energy and width of the antiresonances in an independent fashion. When the number of attached QD's is large, a rich phenomenology appears for different values of the Fermi energy. 
When the Fermi energy lies far from the center of the QW band $\left(\left|\varepsilon-\varepsilon_{0}\right|>2 V_{c}\right)$, the conductance presents regular and smooth behavior. However, the conductance strongly fluctuates close to the center of the QW band $\left(\left|\varepsilon-\varepsilon_{0}\right|<2 V_{c}\right)$. These results pose a question about the relevance of the bifurcation at $\left|\varepsilon-\varepsilon_{0}\right|=2 V_{c}$ in actual experiments. Finally, we found an odd-even parity behavior of the conductance when the Fermi energy lies in center of the band. If the number of QD's in the array is even, perfect transmission takes place $\left(G=2 e^{2} / h\right)$. On the contrary, perfect reflection occurs when this number is odd $(G=0)$. This property arises from the intrinsic electronic properties of the QD array.

We expect that the above picture remains valid even if the electron-electron interaction is taken into account. In fact, in embedded QD arrays, the main effect of the electron-electron interaction is to shift and to split the resonance positions. ${ }^{13,14}$ This occurs because the on-site Coulomb repulsion energy $U$ introduces a renormalization of the site energies. In analogy with embedded QD arrays, we expect that depending on the relation between the interdot coupling and the on-site Coulomb interaction different regimes arise. For $t_{c} / U \ll 1$, the resonances and antiresonances would split into two distinct minibands separated by the on-site Coulomb energy, while for $t_{c} / U \gg 1$, the resonances and antiresonances would occur in pairs. Moreover, we think that the odd-even parity in the conductance also holds if the electron-electron interaction is added. This assumption is based on the fact that the conductance in Hubbard chains coupled to reservoirs shows this odd-even parity. ${ }^{6}$ Therefore, we believe that the interaction would not break this symmetry.

In summary we studied a noninteracting QD array side coupled to a quantum wire. We found that conductance at zero temperature develops an oscillating band with resonances and antiresonances due to constructive and destructive interference in the ballistic channel, respectively. We show that this band is related to the electronic properties of the isolated QD array.

\section{ACKNOWLEDGMENTS}

P.A.O. and I.G. would like to thank Milenio ICM P99135-F and FONDECYT for financial support under Grant No. 1020269. Work in Madrid was supported by DGIMCyT (Grant No. MAT2000-0734) and CAM (Grant No. 07N/ 0075/2001). I.G. would like to thank UCN for the warm hospitality during his visit. M. L. L. d. G. was supported by Cátedra Presidencial en Ciencia F. Claro.
${ }^{1}$ D. Goldhaber-Gordon, H. Shtrikman, D. Mahalu, D. AbuschMagder, U. Meirav, and M. A. Kastner, Nature (London) 391, 156 (1998); D. Goldhaber-Gordon, J. Göres, M. A. Kastner, H. Shtrikman, and D. Mahalu, Phys. Rev. Lett. 81, 5225 (1998).

${ }^{2}$ S. M. Cronenwett, T. H. Oosterkamp, and L. P. Kouwenhoven, Science 281, 5 (1998).

${ }^{3}$ A. W. Holleitner, C. R. Decker, H. Qin, K. Ebert, and R. H. Blick, Phys. Rev. Lett. 87, 256802 (2001).

${ }^{4}$ A. W. Holleitner, R. H. Blick, A. K. Huttel, K. Eber, and J. P. Kotthaus, Science 297, 70 (2002).

${ }^{5}$ W. Z. Shangguan, T. C. Au Yeung, Y. B. Yu, and C. H. Kam, Phys. Rev. B 63, 235323 (2001).
${ }^{6}$ Akira Oguri, Phys. Rev. B 63, 115305 (2001).

${ }^{7}$ Z. Y. Zeng and F. Claro, Phys. Rev. B 65, 193405 (2002).

${ }^{8}$ Tae-Suk Kim and S. Hershfield, Phys. Rev. B 65, 214526 (2002).

${ }^{9}$ B.-Y. Gu, Phys. Rev. B 51, 16840 (1995).

${ }^{10}$ P. S. Deo and A. M. Jaynnavar, Phys. Rev. B 50, 11629 (1994).

${ }^{11}$ J.-R. Shi and B.-Y. Shu, Phys. Rev. B 55, 4703 (1997).

${ }^{12}$ H. S. Wall, Analytic Theory of Continued Fractions (AMS Chelsea, New York, 1967).

${ }^{13}$ G. Chen, G. Klimeck, S. Datta, G. Chen, and W. A. Goddard III, Phys. Rev. B 50, 8035 (1994).

${ }^{14}$ Z. Yu, A. T. Johnson, and T. Heinzel, Phys. Rev. B 58, 13830 (1998). 